\title{
Reward devaluation disrupts latent inhibition in fear conditioning
}

\author{
Luís Gonzalo De la Casa ${ }^{1}$ - Auxiliadora Mena ${ }^{1}$. Juán Carlos Ruiz-Salas ${ }^{1}$. \\ Esperanza Quintero $^{1} \cdot$ Mauricio R. Papini $^{2}$
}

Published online: 11 July 2017

(C) Psychonomic Society, Inc. 2017

\begin{abstract}
Three experiments explored the link between reward shifts and latent inhibition (LI). Using consummatory procedures, rewards were either downshifted from $32 \%$ to $4 \%$ sucrose (Experiments 1-2), or upshifted from $4 \%$ to $32 \%$ sucrose (Experiment 3 ). In both cases, appropriate unshifted controls were also included. LI was implemented in terms of fear conditioning involving a single tone-shock pairing after extensive tone-only preexposure. Nonpreexposed controls were also included. Experiment 1 demonstrated a typical LI effect (i.e., disruption of fear conditioning after preexposure to the tone) in animals previously exposed only to $4 \%$ sucrose. However, the LI effect was eliminated by preexposure to a $32 \%$-to- $4 \%$ sucrose devaluation. Experiment 2 replicated this effect when the LI protocol was administered immediately after the reward devaluation event. However, LI was restored when preexposure was administered after a 60min retention interval. Finally, Experiment 3 showed that a reward upshift did not affect LI. These results point to a significant role of negative emotion related to reward devaluation in the enhancement of stimulus processing despite extensive nonreinforced preexposure experience.
\end{abstract}

Luís Gonzalo De la Casa

delacasa@us.es

1 Department of Experimental Psychology, University of Seville, Sevilla, Spain

2 Department of Psychology, Texas Christian University, Fort Worth, TX, USA
Keywords Latent inhibition · Reward devaluation · Consummatory successive negative contrast · Fear conditioning

Although latent inhibition (LI) was first described in the late 1950s (Lubow \& Moore, 1959), it still continues to command interest. Despite its apparent simplicity and generality across situations, there is no universally accepted explanation for its occurrence. The procedure used to induce LI in the animal laboratory is relatively simple: A stimulus is presented repeatedly by itself before being paired as a conditioned stimulus (CS) with the unconditioned stimulus (US). The result of CSonly presentations is to retard acquisition rate during conditioning, compared to the acquisition rate of a CS that has not been previously exposed by itself (Lubow, 1989).

Despite its apparent simplicity, LI engages associative, attentional, memory, motivational, and emotional processes that make such a phenomenon appealing for an integrative study of the mechanisms participating in stimulus processing (e.g., De la Casa \& Pineño, 2010). Traditionally, most studies on LI have centered on mechanisms related to attention and memory (Lubow \& Weiner, 2010), although there have also been a limited number of studies analyzing the role of emotional factors on LI (e.g., Braunstein-Bercovitz et al. 2001; Hellman, Crider, \& Solomon, 1983; Lazar, Kaplan, Sternberg, \& Lubow, 2012).

In the present experiments, we explored the influence of emotional factors on LI by analyzing the effects of reward downshifts and upshifts on the processing of a stimulus repeatedly presented without specific consequences. As far as we know, there is only one attempt to analyze within a single experiment the effects of inducing positive and negative affect on LI (Lazar et al., 2012). In three experiments with human participants, LI was reduced when the participants had been 
exposed to a negative affect video clip, while LI remained intact when the participants viewed a positive affect video clip before the LI task. These outcomes were interpreted as resulting from an interaction between affect and attention, such that the induction of positive affect would broaden the attentional field (e.g., Fredrickson \& Branigan, 2005), facilitating the decline in associability of the to-be-CS during preexposure, but the induction of negative affect would constrict attention (e.g., Derryberry \& Tucker, 1994), reducing the effect of preexposure to the to-be-CS.

As for evidence from experiments with nonhuman animals, analysis of the role of emotional factors on LI has been mainly centered on the effects of emotional stress (e.g., Hellman et al., 1983; Smith, Fieser, Jones, \& Schachtman, 2008). These studies assumed that stress increases dopamine activity in the nucleus accumbens (e.g., Di Chiara, Loddo, \& Tanda, 1999; Pascucci, Ventura, Latagliata, Cabib, \& Puglisi-Allegra, 2007), and since there is evidence that dopamine hyperactivity in the nucleus accumbens disrupts LI (e.g., Joseph et al., 2000), induced stress should result in LI impairment. However, the results of inducing stress before implementing the LI procedure are far from consistent. As can be seen in Table 1, some experiments have resulted in reduced LI (e.g., McDonald et al., 2002; Smith et al., 2008), while others have produced intact or even enhanced LI (e.g., Hellman et al., 1983; Mongeau, Marcello, Sparre, Andersen, \& Pani, 2007). An inspection of the procedures used to induce stress suggests that those that produce more severe levels of stress (e.g., forced swimming or the combination of tail pressure and amphetamine administration) tended to reduce LI. Conversely, mild levels of stress (e.g., tail pressure or restrained movement) either did not affect or enhanced LI.

In the present experiments, we used consummatory tasks to induce emotional changes before testing for LI. Specifically, to induce a negative emotion we used the reward devaluation task known as consummatory successive negative contrast (cSNC), and to induce a positive emotion we used the reward upshift task named consummatory successive positive contrast (cSPC; Flaherty, 1996).

cSNC involves a downshift in the concentration of a sucrose solution, typically from $32 \%$ to $4 \%$ sucrose. Such a devaluation results in a transient suppression of consummatory behavior compared to an unshifted control always exposed to $4 \%$ sucrose. This effect is accompanied by emotional activation, as suggested by the pharmacological and neurobiological profile typical of situations involving negative emotions (Ortega, Solano, Torres, \& Papini, 2017; Papini, Fuchs, \& Torres, 2015; Papini, Wood, Daniel, \& Norris, 2006; Torres \& Papini, 2016). For example, among other effects, cSNC is modulated by opioids (Papini, 2009), reduced by benzodiazepine anxiolytics (Ortega et al., 2014), it induces postsession preference for oral voluntary consumption of anxiolytics such as ethanol and chlordiazepoxide (Manzo, Donaire, Sabariego,
Papini, \& Torres, 2015), it is reduced by local inactivation in the centromedial amygdala (Kawasaki, Glueck, Annicchiarico, \& Papini, 2015), eliminated by excitotoxic lesions of the basolateral amygdala (Kawasaki, Annicchiarico, Glueck, Morón, \& Papini, 2017), and it leads to enhanced hypothalamic-pituitary-adrenal axis activation (Mitchell \& Flaherty, 1998; Pecoraro, de Jong, \& Dallman, 2009). Based on this profile, and considering the hypothesis that negative emotions constrict the attentional field (e.g., Lazar et al., 2012), we anticipated that reward devaluation would enhance the processing of a stimulus presented immediately after the downshift event, thus reducing the LI effect.

cSPC involves an upshift in the concentration of a sucrose solution. Unlike reward devaluations, reward upshifts are noted by their inconsistent results. Although they have been occasionally reported in the consummatory task (Flaherty, Becker, \& Checke, 1983), these results have not always been replicated (Annicchiarico et al., 2016). For our purposes, however, whether a reward upshift induces cSPC or not, it provides an assessment of the extent to which a change in reward conditions is sufficient to attenuate the LI effect.

We conducted three experiments designed to study the influence of reward downshift and upshift on the processing of a CS after nonreinforced preexposure, assessing the effects in terms of the acquisition of freezing in a fear conditioning situation. In Experiment 1, animals exposed to a $32 \%$-to- $4 \%$ sucrose devaluation underwent LI training in fear conditioning immediately after the devaluation session. In Experiment 2, we predicted that the introduction of a temporal delay between the end of the devaluation event and the initiation of preexposure training in the LI paradigm would eliminate the effect of reward devaluation on LI. In Experiment 3, animals received a 4\%-to$32 \%$ sucrose upshift before the LI treatment, and we predicted a facilitation in processing of the to-be-CS, resulting in an intact or even enhanced LI effect.

\section{Experiment 1}

Groups exposed to either reward devaluation or unshifted controls in Phase 1 were then given either preexposure (PE) or a nonpreexposure (NPE) treatments in a $2 \times 2$ factorial design. Immediately after the last session in the cSNC procedure, animals were exposed in succession to (1) either CSonly trials (PE condition) or an equivalent time in the context (NPE condition), (2) a pairing between the CS and a single footshock, and (3) a 180-s presentation of the CS to assess the strength of conditioning. Attending to the mixed results obtained when a stress-inducing treatment was programmed before testing LI (see Table 1), there are no grounds to make a clear prediction on the effect of the reward devaluation treatment on LI. However, and considering that reward downshift 
Table 1 Review of literature on LI and stress

\begin{tabular}{llll}
\hline Reference & Stress procedures & LI procedure & Effect on LI \\
\hline Hellman et al., 1983 & Tail-pressure & 2-way avoidance & Intact LI \\
& Tail-pressure + amphetamine & & Reduced LI \\
Lehmann et al., 2000 & Prenatal stress & Fear conditioning & Intact LI \\
& Postnatal maternal separation & 2-way avoidance & Enhanced LI \\
McDonald et al., 2002 & Chronic intense noise exposure & Fear conditioning & Reduced LI \\
Melo et al., 2003 & Chronic stress & Fear conditioning & Enhanced LI \\
Mongeau et al., 2007 & Chronic restrain & Fear conditioning & Enhanced LI \\
Shalev et al., 1998 & Acute and chronic administration of corticosterone & Fear conditioning & Reduced LI \\
Smith et al., 2008 & Forced swimming & Conditioned taste aversion & Reduced LI \\
\hline
\end{tabular}

induces a negative emotional state related to the devaluation of reward size (e.g., Manzo et al., 2015; Papini \& Dudley, 1997), and a negative emotional state has been related to LI reduction (e.g., Hellman et al., 1983; Lazar et al., 2012; Smith et al., 2008), we predicted that unshifted PE animals would exhibit less fear conditioning than unshifted NPE animals (i.e., the LI effect), but downshifted PE and downshifted NPE would exhibit just as much conditioning as unshifted NPE animals (i.e., reward devaluation would eliminate the LI effect).

\section{Method}

Subjects Thirty-two male Wistar rats, bread at the University of Seville, served as subjects. The mean weight was $332 \mathrm{~g}$ (range: 278-404 g). Animals were individually housed and maintained on a 12:12 h light:dark cycle (lights on at 06:00 h). All behavioral testing was conducted during the light period of the cycle. Four days before the start of the experiment, each rat was handled $5 \mathrm{~min}$ daily. The day before the start of the experiment, the ad lib weight of each rat was registered, and the food was removed. The food available during the duration of the experiment was reduced to 5-10 g per day; this caused a $15 \%$ drop in ad lib weight by the end of the experiment. All the procedures described in this article were conducted in accordance with the guidelines established by Directive $86 / 609 / \mathrm{CEE}$ of the European Community Council and the Spanish R.D. 223/1988.

Apparatus Four identical Panlab conditioning boxes (Model LE111, Panlab/Harvard Apparatus, Spain) were used, each measuring $26 \mathrm{~h} \times 25 \mathrm{l} \times 25 \mathrm{w} \mathrm{cm}$. Each chamber was enclosed in a sound-attenuating cubicle (Model LE116. Panlab/Harvard Apparatus, Spain). The walls of the experimental chambers were made of white acrylic. Solutions were prepared $w / v$ by dissolving $4 \mathrm{~g}$ ( or $32 \mathrm{~g}$ ) of sucrose for every liter of tap water for $4 \%$ (and 32\%) solutions. They were presented at room temperature in 150-ml bottles with stainless steel spouts attached to the front of each conditioning box $(6 \mathrm{~cm}$ from the floor). Consumption was calculated on each session by weighing the bottles before and after fluid presentation to the nearest $0.05 \mathrm{~g}$ using a compact scale (Mettler Toledo BPA224-1.5AP, Spain). Session length was measured with a manual stopwatch (Amarell Electronic Timer-Clock, Germany).

A loudspeaker located at the top of each chamber produced a $70 \mathrm{~dB} 2.8-\mathrm{kHz} 30$-s tone used as the CS. The floor in each chamber consisted of stainless steel rods, $2 \mathrm{~mm}$ in diameter, spaced $10 \mathrm{~mm}$ apart (center to center). The US was a 1-sec, 0.5-mA unscrambled AC 50-Hz footshock from a constantcurrent generator (Model LE100-26, Panlab/Harvard Apparatus, Spain) delivered to the floor of each chamber that rested on a platform that recorded the signal generated by the animal movement through a high sensitivity weight transducer system. Such a signal was automatically converted into a percentage of general activity, defined as the percentage of the total time that movement was detected during a CS presentation (PE condition) or in a period of the same length (NPE condition), by a commercial software (StartFear system software, Panlab/Harvard Apparatus, Spain). Sampling was performed continuously at a frequency of $50 \mathrm{~Hz}$.

Procedure Sessions 1-10 were preshift sessions in the cSNC task. Half the animals received $5 \mathrm{~min}$ of access to $4 \%$ sucrose and the other half to $32 \%$ sucrose. On Session 11, all animals received $5 \mathrm{~min}$ of access to $4 \%$ sucrose. Thus, for half the animals, this involved a 32\%-to-4\% sucrose downshift, whereas for the other half the concentration did not change from the previous session. Immediately after the 5-min downshift trial, the bottles were removed from the conditioning boxes and the LI protocol was initiated. Thus, animals stayed in the same conditioning boxes during this session.

In LI, half of the animals previously receiving either $32 \%$ or $4 \%$ sucrose were preexposed to $25 \mathrm{CS}$-only presentations (PE condition). The first tone CS was presented after a 300-s period without stimulation. Each CS lasted $30 \mathrm{~s}$. The intertrial interval was $30 \mathrm{~s}$ on average (range: $20-40 \mathrm{~s}$ ). The other half of each contrast condition were exposed to the context only 
(NPE condition). These animals remained an equivalent period of time in the experimental chambers without any additional stimulation. A 30-s period after the last CS-only presentation (PE condition) or the equivalent context-only exposure (NPE condition) preceded the only conditioning trial. In this trial, the CS was presented for $30 \mathrm{~s}$, and its termination coincided with the delivery of an electric footshock ( $1 \mathrm{~s}, 0.5 \mathrm{~mA})$. This conditioning trial was followed by a 180 -s interval that ended with the single test trial. This final test trial consisted of a 180-s tone-alone trial similar for all the animals. The total duration of the session was approximately $40 \mathrm{~min}$.

The dependent variables were the amount of sucrose consumed (ml) during cSNC sessions and the percentage of general activity recorded during tone presentations (or during an equivalent period for the animals in the NPE condition) collapsed across five trials. Activity during the CS at testing was considered an index of fear conditioning, with lower levels of activity reflecting higher levels of fear. All the results presented in this article were analyzed with the IBM SPSS Statistics v. 24 package, with an alpha level set at 0.05 , and with LSD pair-wise comparisons derived from the main analysis.

\section{Results}

Figure 1, top panel, depicts sucrose consumption as a function of concentration $(32 \%, 4 \%)$. Consumption increased across preshift Sessions 1-10 more for groups that had access to $32 \%$ sucrose than for groups that received $4 \%$ sucrose. A Contrast $(32 \%, 4 \%) \times$ Preexposure $(\mathrm{PE}, \mathrm{NPE}) \times$ Session $(1-$ 10) analysis indicated a significant contrast by session interaction, $F(9,252)=4.50, p<.001$, and significant main effects for contrast and sessions, $F \mathrm{~s}>10.04, p \mathrm{~s}<.001$. Other effects were nonsignificant, $F_{\mathrm{S}}<1.37, p \mathrm{~s}>.20$. Thus, there was no evidence of a bias in terms of assignment to the preexposure conditions, as none of the effects involving this factor were significant. The results of the single downshift session, Session 11, are also presented in Fig. 1, top panel. Despite the small size of the difference, there was a significant cSNC effect as revealed for the reduced sucrose consumption for those animals in the 32-4 as compared to those in the 4-4 condition, $F(1,28)=4.82, p<.04$. Other effects were nonsignificant, $F_{\mathrm{S}}<1.21, p \mathrm{~s}>0.28$, again pointing to the absence of bias as far as assignment of animals to the preexposure conditions.

Figure 1, middle panel, shows the results of preexposure trials. During preexposure to the $\mathrm{CS}$, there was a significant interaction between the preexposure condition (PE, NPE) and blocks of trials, $F(1,28)=5.77, p<.03$. There was also a significant reduction in activity across preexposure blocks, $F(4,112)=21.90, p<.001$. Other factors were not significant, $F_{\mathrm{s}}<2.70, p s>.11$. The preexposure by blocks interaction was due to a relatively high activity level in the $32-4 / \mathrm{PE}$ condition during the last two blocks of preexposure.
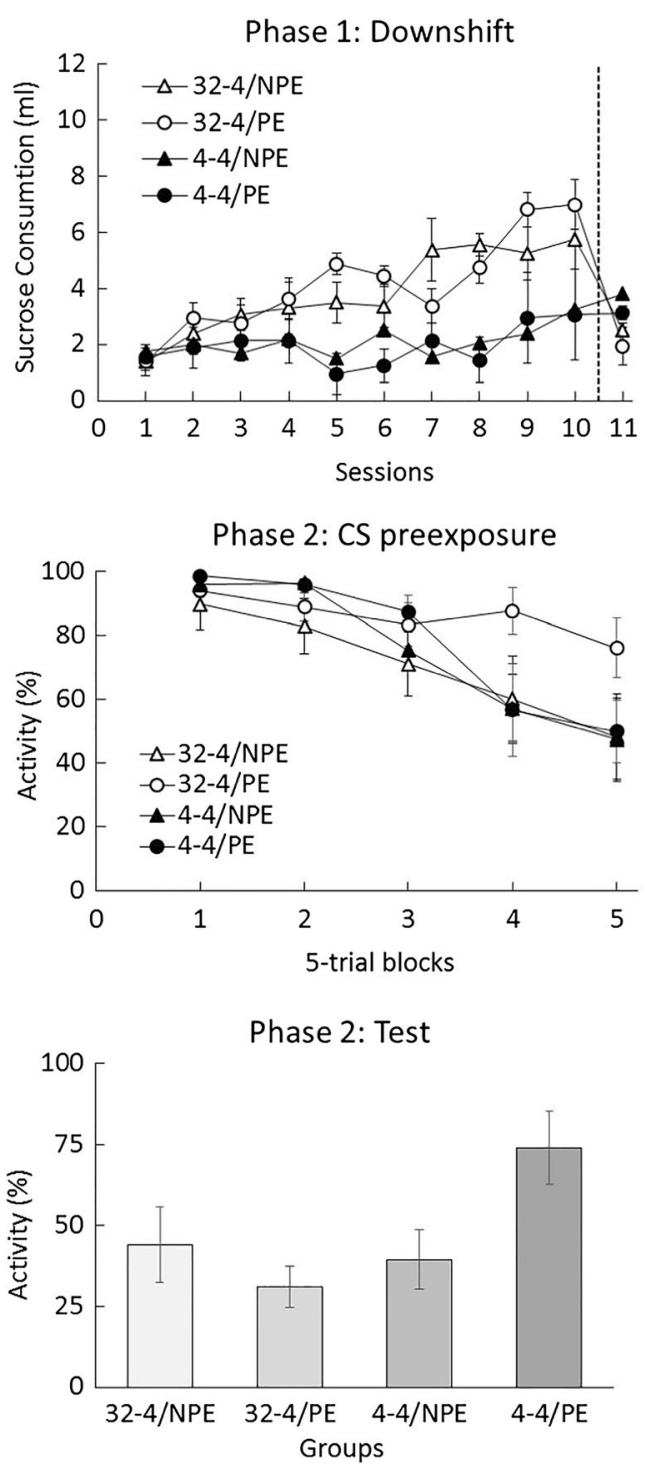

Fig. 1 Top panel: Mean (SEM) sucrose consumption (ml) as a function of sucrose concentration $(32 \%, 4 \%)$ during preshift sessions (1-10) and the only downshift session (11). Middle panel: Activity during preexposure trials. Bottom panel: Activity during the test session for fear conditioning. Groups had been previously exposed (PE) or not (NPE) to a tone to be used as the CS in the fear conditioning task

The main results from the LI test are presented in Fig. 1, bottom panel. The analysis indicated a significant preexposure by contrast interaction, $F(1,28)=5.77, p<.03$. The main effects were not significant, $F \mathrm{~s}<3.74$, $p \mathrm{~s}>.06$. Pair-wise LSD comparisons derived from the main analysis showed that Group 4-4/ PE was significantly different than Group 4-4/NPE, $F(1,28)=$ $6.11, p<.03$, thus confirming that rats preexposed to the unshifted reward condition exhibited the LI effect. Importantly, Groups 32-4/PE and 32-4/NPE did not differ from each other, $F<1$, thus suggesting that the reward downshift experience eliminated the LI effect in fear conditioning. In addition, Group 4-4/PE displayed significantly more activity than Group 32-4/PE, $F(1,28)=9.39, p<.006$, whereas Groups 4-4/ NPE and 32-4/NPE were not different from each other, $F<1$. 
Thus, the reward downshift experience counteracted the effects of CS preexposure on fear conditioning.

An additional analysis was conducted on mean activity during the $180 \mathrm{~s}$ that preceded CS presentation (pre-CS period) and followed the single CS-US pairing in the final test because it is possible that the devaluation treatment could have selectively affected activity independently of the CS, thus complicating the interpretation of the main results. Group means are presented in Table 2. A Preexposure $\times$ Contrast analysis of these pre-CS data revealed a significant interaction, $F(1,28)=13.03, p<.002$. There was also a main effect of contrast, $F(1,28)=5.59, p<.03$, due to a higher level of activity in groups given access to $4 \%$ sucrose than to $32 \%$ sucrose. The main effect of preexposure was nonsignificant, $F$ $<1$. Finally, LSD pair-wise comparisons derived from the main analysis revealed reduced activity in Group 32-4/PE compared to Groups 32-4/NPE and 4-4/PE, $F \mathrm{~s}(1,28)>$ $6.39, p<.02$. In addition, Group 4-4/NPE also exhibited reduced activity relative to $4-4 / \mathrm{PE}, F(1,28)=6.63, p<.02$. These data, and particularly the reduced activity observed in Group 32-4/PE compared to Group 32-4/NPE, compromise the interpretation of CS data during the final test. Apparently, reward downshift reduced activity both in the presence and absence of the CS during the final test.

\section{Experiment 2}

One goal of this experiment was to reproduce the conditions that apparently disrupted LI in Experiment 1 to determine whether the result observed in Group 32-4/PE reflects a selective reduction of activity to the CS or a general reduction of activity during both CS and pre-CS periods.

Additionally, this experiment was designed to test a specific prediction derived from studies of reward devaluation that suggest the effect tends to decay in time, in the order of seconds to minutes after the downshift event. For example, rats trained in Pavlovian and instrumental analogues of the double-runway frustration effect (Amsel \& Roussell, 1952) exhibit response invigoration immediately after a surprising reward omission, but the effect dissipates when the opportunity to respond is delayed for 20 to 80 s (Dudley \& Papini, 1995; Papini \& White, 1994; Stout, Boughner, \& Papini, 2003). In the cSNC situation, interpolating an interval of 8 days between Sessions 11 and 12 eliminated the effect (Ortega et al., 2014). However, nothing is known about the effect on the cSNC situation of interpolating shorter retention intervals while remaining in the same context, as was done in the present experiment. Animals were preexposed to the CS and later conditioned and tested for LI within the same session in which they had experienced reward downshift. If the elimination of LI after reward devaluation described in Experiment 1 was related to frustration, then the effect should be replicated when tested immediately after the downshift, but not when preexposure training was delayed by $60 \mathrm{~min}$. Additionally, if the effect was the result of a nonselective reduction of general activity, then activity should be reduced not only during CS presentation but also during the pre-CS period in groups replicating the results of the previous experiment.

\section{Method}

Subjects and apparatus Forty male Wistar rats bred at the University of Seville, Spain, were used (mean weight: $449 \mathrm{~g}$, range: $328-584 \mathrm{~g}$ ). Housing, maintenance, and the training equipment were as described in Experiment 1.

Procedure Consummatory training and LI testing were as described for Groups 32-4/PE and 32-4/NPE, in Experiment 1, except for the following. Once the 5-min downshift session (Session 11) ended, animals were returned to their home cage, and the bottles were removed from the conditioning boxes. Half the subjects were immediately returned to the conditioning boxes, and the LI protocol described in Experiment 1 was initiated. The other half remained in their home cage for 1 hour before being returned to the conditioning boxes to receive the LI protocol already described. Animals were randomly assigned to four groups $(n=10)$ : 0/PE, 60/PE, 0/NPE, and 60/NPE. Thus, all animals received $32 \%$ sucrose for 10 sessions followed by $4 \%$ sucrose on Session 11, they were placed in their home cage and returned to the conditioning box either immediately or after $60 \mathrm{~min}(0,60)$, and they received either preexposure or no preexposure as part of the LI treatment (PE, NPE). Other aspects of the procedure were as described in Experiment 1.

Table 2 Mean percentage activity during the pre-CS period

\begin{tabular}{lllll}
\hline Exp. 1 & $32-4 / \mathrm{NPE}$ & $32-4 / \mathrm{PE}$ & $4-4 / \mathrm{NPE}$ & $4-4 / \mathrm{PE}$ \\
Mean $(S E M)$ & $72.40(8.78)$ & $47.83(7.72)$ & $63.84(4.37)$ & $88.86(5.76)$ \\
Exp. 2 & $32-4 / 0 / \mathrm{NPE}$ & $32-4 / 0 / \mathrm{PE}$ & $32-4 / 60 / \mathrm{NPE}$ & $32-4 / 60 / \mathrm{PE}$ \\
Mean (SEM) & $17.47(4.75)$ & $21.28(5.42)$ & $14.77(4.24)$ & $20.45(5.00)$ \\
Exp. 3 & $4-32 / \mathrm{NPE}$ & $4-32 / \mathrm{PE}$ & $32-32 / \mathrm{NPE}$ & $32-32 / \mathrm{PE}$ \\
Mean $(S E M)$ & $60.61(14.11)$ & $54.76(34.58)$ & $75.85(14.29)$ & $65.28(23.11)$ \\
\hline
\end{tabular}




\section{Results}

Figure 2, top panel, shows the results of the cSNC phase of the experiment. All animals increased sucrose intake across sessions, $F(9,324)=65.04, p<.001$. There was also a significant interaction between retention interval and session, $F(9,324)=$ $2.67, p<.006$. This interaction is detecting the crossing over of groups, especially on Session 9, and it was therefore not given much weight. Other effects were nonsignificant, $F_{\mathrm{S}}<$ $1.78, p s>0.19$. Sucrose consumption decreased sharply on Session 11. Although there was a tendency for animals that were to receive NPE treatment to decrease their intake more than those that were to receive PE treatment, the difference did
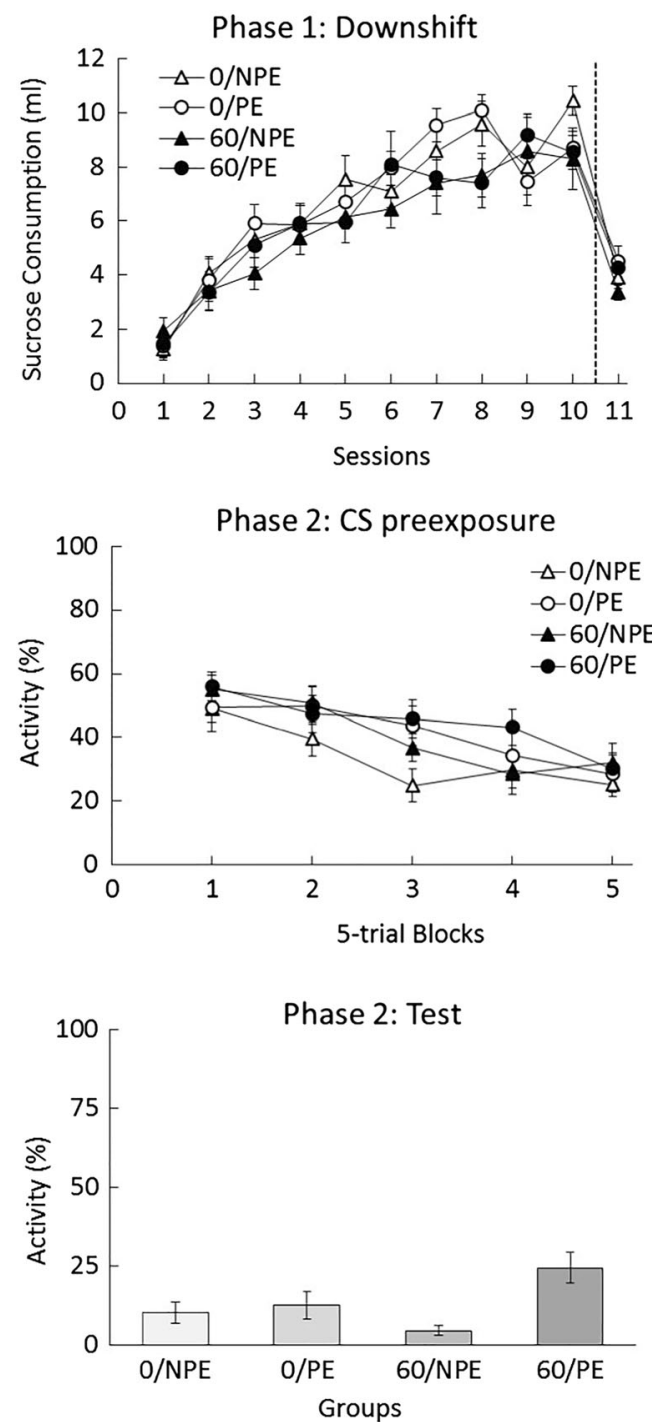

Fig. 2 Top panel: Mean (SEM) 32\% sucrose consumption (ml) during preshift sessions (1-10) and $4 \%$ sucrose consumption during one downshift session (11). Middle panel: Activity during preexposure trials. Bottom panel: Activity in a test trial after preexposure (PE) or not (NPE), followed by a single fear conditioning trial. Groups differed in terms of a retention period between reward downshift and preexposure treatment, either immediate (0) or delayed by $60 \mathrm{~min}(60)$ not reach significance, $F(1,36)=3.43, p>.07$. Other effects were nonsignificant, $F_{\mathrm{S}}<1$.

There was a general reduction of activity during preexposure training in Experiment 2 compared to Experiment 1 (compare the middle panels of Figs. 1 and 2). The reason for these differences is unclear, since the procedures that were followed were identical, with one major exception. In Experiment 1, after the downshift trial, animals remained undisturbed in the conditioning boxes until the LI treatment was initiated. However, in Experiment 2, each rat was removed from the conditioning box and introduced again to receive the LI treatment after zero or $60 \mathrm{~min}$. It is likely that such manipulation increased general levels of freezing. In spite of this circumstance, the results were clear: During preexposure there was again a preexposure by session effect, $F(4,144)=2.65, p<.04$, mostly due to Group 0/NPE, which displayed low activity levels during the third trial block. There was also a significant reduction in activity across sessions, $F(4,144)=27.95, p<.001$. All other factors were not significant, $F \mathrm{~s}<1.70, p \mathrm{~s}>.15$.

Figure 2, bottom panel, shows the main results of this experiment. A Preexposure $(\mathrm{NPE}, \mathrm{PE}) \times$ Retention Interval $(0$, $60 \mathrm{~min}$ ) analysis showed a significant interaction, $F(1,36)=$ $5.48, p<.03$, and also a significant main effect of preexposure, $F(1,36)=8.60, p<.007$. The main effect of the retention interval condition was not significant, $F<1$. LSD pair-wise tests derived from this analysis indicated that activity in Group 60/PE was significantly higher than in Group 60/NPE, $F(1,36)=13.91, p<.002$, a result demonstrating the LI effect after a 60 -min retention interval. Group 60/PE was also significantly different from Group 0/PE, $F(1$, $36)=5.11, p<0.04$, indicating that the reward devaluation event erased the effects of preexposure on fear conditioning when there was a minimal retention interval. That there was no evidence of a LI effect after the short retention interval is indicated also by a nonsignificant difference between Groups $0 / \mathrm{NPE}$ and $0 / \mathrm{PE}, F<1$.

To determine whether the effect of reward devaluation on LI was related to CS processing during preexposure or to a general reduction of activity, a Preexposure $\times$ Retention Interval analysis was conducted on activity during the preCS period. The means are presented in Table 2. The analysis showed that neither the main effects nor the interaction was significant, $F \mathrm{~S}<1$.

\section{Experiment 3}

The results of Experiment 2 indicated that the reward devaluation treatment had a disruptive effect on LI when it was conducted immediately before CS preexposure, but such effect vanished when a 60-min retention interval was introduced between the devaluation episode and the LI treatment. In addition, the absence of differences on general activity during 
the pre-CS period is consistent with the hypothesis that the reduction of LI in animals exposed to reward devaluation was caused by the emotional effect induced by the devaluation treatment.

In this experiment, we reproduced the same design used in Experiment 1, but the key group was exposed to a 4\%-to-32\% sucrose upshift. There was also an unshifted control always receiving 32\% sucrose. Both groups received the LI treatment immediately after the last contrast session. Since reward upshift should induce a positive emotional state, and such states have been shown to leave LI intact (Lazar et al., 2012), we predicted that the LI effect would remain intact or even be enhanced for upshifted PE animals. In addition, introducing a reward upshift before the LI treatment will allow testing an explanation of the reduced LI found in Experiments 1 and 2 based on reward changes. It is possible that any change in reward would cause increase attention because of its surprising nature. There is evidence that a surprising event can restore the associability of a previously preexposed CS (e.g., Hall \& Pearce, 1982). Therefore, if the reduced LI effect observed in previous experiments was the consequence of the surprise produced by the reward change, we should find a similar reduction of LI in this experiment because the 4\%to-32\% sucrose change should be just as surprising as the $32 \%$-to- $4 \%$ sucrose change.

\section{Method}

Subjects and apparatus Thirty-two male Wistar rats bred at the University of Seville, Spain, were used (mean weight: $340 \mathrm{~g}$, range: $291-450 \mathrm{~g}$ ). Housing, maintenance, and the training equipment were as described in Experiment 1.

Procedure Sessions 1-10 were preshift sessions in the cSPC task. A random half of the animals received $5 \mathrm{~min}$ of access to $4 \%$ sucrose and the other half to $32 \%$ sucrose. On Session 11, all animals received $5 \mathrm{~min}$ of access to $32 \%$ sucrose. Thus, for half the animals, this involved a 4\%-to-32\% sucrose change, whereas for the other half the concentration was always $32 \%$. Immediately after this 5-min trial, the bottles were removed from the conditioning boxes, and the same LI protocol described in Experiment 1 was initiated.

\section{Results}

Figure 3, top panel, shows the results of the reward upshift manipulation. The top panel depicts sucrose consumption as a function of concentration. Consumption increased across preshift Sessions 1-10 more for groups that had access to $32 \%$ sucrose than for groups that received $4 \%$ sucrose. A Contrast $(32 \%, 4 \%) \times$ Preexposure $(\mathrm{PE}, \mathrm{NPE})$ $\times$ Session (1-10) analysis indicated a significant contrast by session interaction, $F(9,252)=3.05, p<.003$, and
Phase 1: Upshift

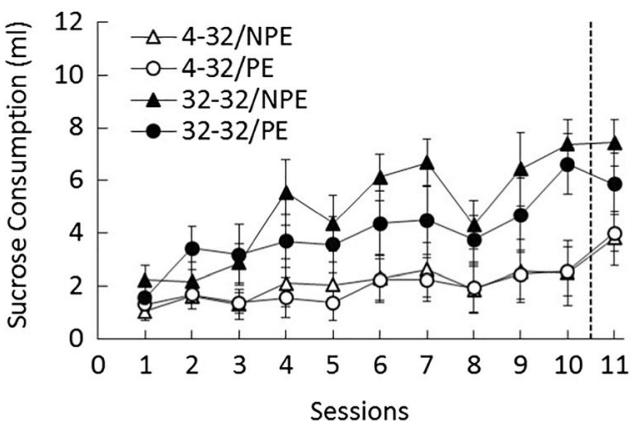

Phase 2: CS preexposure
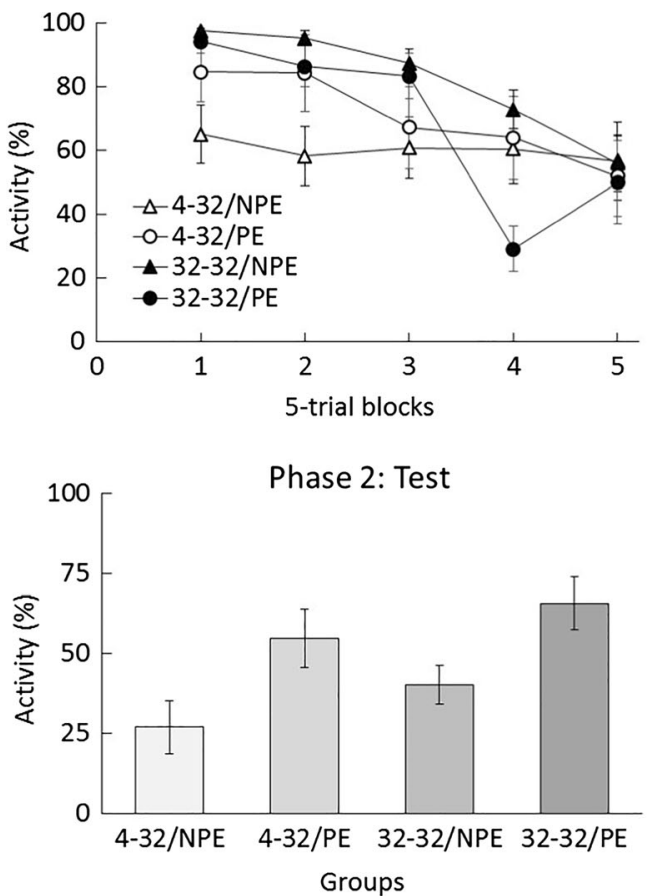

Fig. 3 Top panel: Mean (SEM) sucrose consumption (ml) as a function of sucrose concentration $(32 \%, 4 \%)$ during preshift sessions (1-10) and the only upshift session (11). Middle panel: Activity during preexposure trials. Bottom panel: Activity during the test session for fear conditioning. Groups had been previously exposed (PE) or not (NPE) to a tone to be used as the CS in the fear conditioning task

significant main effects for contrast and sessions, $F \mathrm{~s}>$ 10.23, $p \mathrm{~s}<.004$. The remaining effects and interactions were nonsignificant, $F \mathrm{~s}<1.01 . p \mathrm{~s}>.43$. The results of the single upshift session are also presented in Fig. 3, top panel. The main effect of contrast was significant as revealed by the increase in sucrose consumption from Session 10 to Session 11 for those animals in the 4-32 groups, $F(1,28)=$ $8.20, p<.009$, but notice that in this case upshifted groups were significantly below unshifted controls - the opposite of a cSPC effect. Similar results have been reported in analogous experiments involving reward upshifts (Annicchiarico et al., 2016). Other effects were nonsignificant, $F \mathrm{~S}<1$, pointing to the absence of bias as far as assignment of animals to the preexposure conditions. 
Preexposure data are presented in Fig. 3, middle panel. During preexposure to the CS, there was a significant reduction in activity across preexposure sessions, $F(4,112)=20.56$, $p<.001$. Also, the interactions of preexposure and contrast with blocks were both significant, $F \mathrm{~s}(4,112)>3.50, p \mathrm{~s}<.02$. Other factors and interactions were not significant, $F_{\mathrm{S}}<2.11$, $p \mathrm{~s}>.08$. LSD pair-wise comparisons confirmed that the blocks by preexposure interaction was due to a relatively low activity in PE groups (driven exclusively by Group 32$32 / \mathrm{PE})$ on Block $4, F(1,28)=4.24, p<.05$. The Blocks $\times$ Contrast interaction was due to higher activity during the first three blocks in animals previously exposed to the 4\%-to-32\% sucrose upshift, $F \mathrm{~s}>5.48, p \mathrm{~s}<.03$.

Figure 3, bottom panel, shows mean percentage of activity during the CS presentation in the final test. A Preexposure $\times$ Contrast analysis revealed that $\mathrm{PE}$ groups displayed higher CS activity than NPE groups, $F(1,28)=10.94, p<.004$, due to an overall LI effect. The contrast and interaction effects were not significant, $F_{\mathrm{s}}(1,28)<2.32, p \mathrm{~s}>.12$.

The bottom section of Table 2 shows mean percentage of activity during the pre-CS period. A Preexposure (NPE, PE) $\times$ Contrast (4-32, 32-32) analysis indicated that none of the factors achieved significance, $F_{\mathrm{s}}(1,28)<2.49, p \mathrm{~s}>.12$.

\section{General discussion}

These experiments showed that nonreinforced preexposure to the CS immediately after a reward devaluation event eliminated the LI effect; groups given unshifted access to the reward exhibited a regular LI effect (Experiment 1). In addition, the effects of reward devaluation were transient, as indicated by the emergence of the LI effect when a 60-min retention interval was interpolated between reward devaluation and the preexposure treatment (Experiment 2). Finally, there was no evidence that the LI effect was affected by a reward upshift treatment (Experiment 3), suggesting that the effect on LI described in Experiments 1 and 2 was due to reward devaluation rather than just a change in reward conditions.

The reduced activity observed in Group 32-4/PE in Experiment 1 cannot be considered as evidence of an effect of negative affect on LI selective to the CS, since a reduction of activity also appeared in the pre-CS period specifically for this group (see Table 1). The source of this general effect is difficult to identify. Perhaps, a combination of the negative affect induced by the reward devaluation treatment and the presentation of a novel stimulus (the to-be-CS) resulted in a sensitized fear response to the US (e.g., Rau \& Fanselow, 2009). However, the lack of differences in pre-CS activity between Groups 0/NPE and 0/PE in Experiment 2, both receiving similar treatments to those of Group 32-4/NPE and 32-4/PE in Experiment 1, do not support a sensitization hypothesis.
Indeed, the results of Experiment 2 offered a clear confirmation of our hypothesis that the induction of negative affect by means of reward devaluation would disrupt LI. This result is relevant to at least two problems. First, the role of emotional factors in LI, a learning phenomenon traditionally explored from a cognitive perspective, and second, the links between psychological pain (e.g., induced by reward devaluation, as in the cSNC situation) and physical pain (e.g., induced by peripheral activation of pain receptors, as in fear conditioning).

Regarding the role of emotional factors, as mentioned in the introduction, previous research has revealed that both negative affect (Lazar et al., 2012) and intense emotional stress can disrupt the LI effect (e.g., Shalev, Feldon, \& Weiner, 1998; Smith et al., 2008). Such results have been traditionally interpreted in the context of stress-induced increases in attention to the preexposed stimulus mediated by enhanced dopamine activity in the nucleus accumbens (e.g., McDonald et al., 2002). In Amsel's (1992) frustration theory, surprising reward omissions or devaluations have both associative and nonassociative effects. Associative effects involved the acquisition of an anticipatory frustration state that promotes avoidance of the goal. More related to the present experiments, surprising nonreward also induces an increase in drive that presumably underlies response invigoration (Stout et al., 2003). Drive induction is a concept that can be reframed as attentional enhancement, a conceptual shift that might close the circle in an understanding of the effects of reward devaluation on LI observed in the present experiments.

The attentional perspective of LI that attributes a weak CSUS association to a reduction in attention to a preexposed CS (Lubow, 1989; Pearce \& Hall, 1980) contrasts with an alternative view proposing that different associations are established during the two stages of a LI experiment: A CSnothing association acquired during nonreinforced presentations of the CS and a CS-US association established during conditioning trials. At the time of testing, both associations would compete for behavioral expression, inducing the reduced conditioned response that characterizes LI, a competition that would be attenuated or absent in the nonpreexposed group (Bouton, 1993). Considering that memory retrieval is impaired by stress (e.g., Park, Zoladz, Conrad, Fleshner, \& Diamond, 2008; Rashidy-Pour, Sadeghi, Taherain, Vafaei, \& Fathollahi, 2004), the reduced LI observed in the present experiments could be interpreted not only as the result of increased attention to the CS during preexposure but also as the result of an impaired CS-nothing association retrieval at the time of testing due to the effects of negative emotion induced by reward devaluation when the CS-nothing association was being acquired. Therefore, the present results do not distinguish between the attentional and the interference theories of LI. A possible alternative explanation to the present results relies on the well-established contextual dependence of LI (e.g., Hall \& Channell, 1986; Lovibond, Preston, \& 
Mackintosh, 1984). More specifically, when preexposure and conditioning stages are conducted in different contexts, LI is impaired whether the change involves the physical context (Westbrook, Jones, Bailey, \& Harris, 2000) or the organism's internal state (Overton, 1974; Wynne \& Delius, 1995), or even when the different experimental stages are conducted at different times during the day (Manrique et al., 2004; MoleroChamizo, 2017). Thus, it is possible that the emotional internal state would differ at the time of preexposure relative to the state during conditioning (programmed approximately $25 \mathrm{~min}$ after the cSNC episode), as the emotion induced by reward devaluation decayed (see Experiment 2). Therefore, the reduced LI effect observed in Groups 32/PE and 0/PE in Experiments 1 and 2, respectively, can be interpreted as the result of a change in the emotional internal context from preexposure to conditioning. Additional research is needed to explore the possible role of change in internal context on LI.

A brief comment on the failure of reward upshift to influence LI is due. In Experiment 3, an equivalent, but opposite, change in reward relative to that implemented in Experiment 1 yielded no evidence of an effect on LI. There are at least two possible accounts that apply to these results. First, it can be argued that the lack of effect on LI was due to inadequate training parameters to induce a cSPC effect during Phase 1. In view of the extensive series of experiments showing difficulties to obtain the cSPC effect, this possibility seems unlikely (Annicchiarico et al., 2016). Second, even if no evidence of the cSPC effect is obtained, this would not necessarily preclude the presence of a positive emotion induced by reward upshift. If this were the case, then one would have to conclude that positive emotions do not influence LI in fear conditioning, either by generating a different internal context or by reestablishing attention to the CS. Clearly, more research is needed to determine whether positive emotions can influence LI.

The present results are also relevant to the interactions between psychological and physical pain (Papini et al., 2015; Papini et al., 2006). The basic assumption is that these two forms of pain share some fundamental mechanisms at the neurochemical and neural levels that allow them to interact in selective ways. For example, exposure to reward devaluation, which is known to induce the release of endogenous opioids (Pellegrini, Wood, Daniel, \& Papini, 2005), leads to reduced sensitivity to peripheral physical pain (JiménezGarcía et al., 2016; Mustaca \& Papini, 2005). Such hypoalgesia could interfere with fear conditioning by reducing the subjective intensity of the electric shock. However, no evidence of such an effect was observed in the present results. For example, for NPE groups, downshifted and unshifted animals exhibited no differences in fear conditioning (Figs. 1 and 2, bottom panels). It is possible that by the time animals were exposed to the shock, the hypoalgesic response was sufficiently attenuated to make little difference. There is evidence of such time decay after a single exposure to reward devaluation (Jiménez-García et al., 2016). The connection between reward devaluation and fear conditioning also deserves additional study.

We started by assuming that several processes underlie the LI effect, including the emotional state at the time of preexposure and testing. The general trend in the literature has been to neglect the role of emotion in the LI effect, irrespective of the procedure used to preexpose the target stimulus. However, a closer look at the typical procedures used to induce LI in the animal literature (e.g., fear conditioning, appetitive conditioning, avoidance learning, conditioned taste aversion) suggests that differences in the emotional state of the animal related, for example, to food deprivation and to the nature of the CS and US, could potentially affect the way in which stimuli are processed (De la Casa, 2013).

Acknowledgments This research was supported by grants from Spanish Ministerio de Economia y Competitividad (PSI2012-32077, and PSI2015-64965-P/MINECO-FEDER, UE).

M. R. Papini's participation was supported in part by a U.S. Scholar Fulbright Award. The authors wish to thank Aaron Fernández for his help in running the experiments.

\section{References}

Amsel, A. (1992). Frustration theory. Cambridge, UK: Cambridge University Press.

Amsel, A., \& Roussell, J. (1952). Motivational properties of frustration: I. Effect on a running response of the addition of frustration to the motivational complex. Journal of Experimental Psychology, 43, 363-368.

Annicchiarico, I., Glueck, A. C., Cuenya, L., Kawasaki, K., Conrad, S. E., \& Papini, M. R. (2016). Complex effects of reward upshift on consummatory behavior. Behavioural Processes, 129, 54-67.

Bouton, M. E. (1993). Context, time, and memory retrieval in the interference paradigms of Pavlovian learning. Psychological Bulletin, 114, 80-99.

Braunstein-Bercovitz, H., Dimentman-Ashkenazi, I., \& Lubow, R. E. (2001). Stress affects the selection of relevant from irrelevant stimuli. Emotion, 1, 182-192.

De la Casa, L. G. (2013). Food and water deprivation disrupts latent inhibition with an auditory fear conditioning procedure. Behavioural Processes, 100, 54-57.

De la Casa, L. G., \& Pineño, O. (2010). Inter-stage context and time as determinants of latent inhibition. In R. E. Lubow \& I. Weiner (Eds.), Latent inhibition: Cognition, neuroscience and applications to schizophrenia (pp. 40-61). Cambridge, UK: Cambridge University Press.

Derryberry, D., \& Tucker, D. M. (1994). Motivating the focus of attention. In P. M. Neidenthal \& S. Kitayama (Eds.), The heart's eye: Emotional influences in perception and attention (pp. 167-196). San Diego, CA: Academic Press.

Di Chiara, G., Loddo, P., \& Tanda, G. (1999). Reciprocal changes in prefrontal and limbic dopamine responsiveness to aversive and rewarding stimuli after chronic mild stress: Implications for the psychobiology of depression. Biological Psychiatry, 46, 1624-1633. 
Dudley, R. T., \& Papini, M. R. (1995). Pavlovian performance of rats following unexpected reward omissions. Learning \& Motivation, 26, 63-82.

Flaherty, C. F. (1996). Incentive relativity. Cambridge, UK: Cambridge University Press.

Flaherty, C. F., Becker, H., \& Checke, S. (1983). Repeated contrast in consummatory and open field behaviors with repeated reward shifts. Animal Learning and Behavior, 11, 407-414.

Fredrickson, B. L., \& Branigan, C. (2005). Positive emotions broaden the scope of attention and thought-action repertoires. Cognition \& Emotion, 19, 313-332.

Hall, G., \& Channell, S. (1986). Context specificity of latent inhibition in taste aversion learning. Quarterly Journal of Experimental Psychology Section B, 38(2), 121-139.

Hall, G., \& Pearce, J. M. (1982). Restoring the associability of a preexposed CS by a surprising event. The Quarterly Journal of Experimental Psychology Section B, 34(3), 127-140.

Hellman, P. A., Crider, A., \& Solomon, P. R. (1983). Interaction of tail pressure stress and $d$-amphetamine in disruption of the rat's ability to ignore an irrelevant stimulus. Behavioral Neuroscience, 97, 10171021.

Jiménez-García, A. M., Ruíz-Leyva, L., Cendán, C. M., Torres, C., Papini, M. R., \& Morón, I. (2016). Hypoalgesia induced by reward devaluation in rats. PloS One, 11(10), e0164331. doi:10.1371/ journal.pone. 0164331

Joseph, M. H., Peters, S. L., Moran, P. M., Grigoryan, G. A., Young, A. M. J., \& Gray, J. A. (2000). Modulation of latent inhibition in the rat by altered dopamine transmission in the nucleus accumbens at the time of conditioning. Neuroscience, 101, 921-930.

Kawasaki, K., Annicchiarico, I., Glueck, A. C., Morón, I., \& Papini, M. R. (2017). Reward loss and the basolateral amygdala: A function in reward comparisons. Behavioural Brain Research, 331, 205-213.

Kawasaki, K., Glueck, A. C., Annicchiarico, I., \& Papini, M. R. (2015). Function of the centromedial amygdala in reward devaluation and open-field activity. Neuroscience, 303, 73-81.

Lazar, J., Kaplan, O., Sternberg, T., \& Lubow, R. E. (2012). Positive and negative affect produce opposing task-irrelevant stimulus preexposure effects. Emotion, 12, 591-604.

Lehmann, J., Stöhr T. h., \& Feldon, J. (2000). Long-term effects of prenatal stress experience and postnatal maternal separation on emotionality and attentional processes. Behavioural Brain Research, 107, 133-144.

Lovibond, P., Preston, G. C., \& Mackintosh, N. J. (1984). Contextual control of conditioning and latent inhibition. Journal of Experimental Psychology: Animal Behavior Processes, 10, 360375.

Lubow, R. E. (1989). Latent inhibition and conditioned attention theory. New York, NY: Cambridge University Press.

Lubow, R. E., \& Moore, A. U. (1959). Latent inhibition: The effect of non-reinforced preexposure to the conditional stimulus. Journal of Comparative and Physiological Psychology, 52, 415-419.

Lubow, R. E., \& Weiner, I. (2010). Latent inhibition: Cognition, neuroscience and application to schizophrenia. Cambridge, UK: Cambridge University Press.

Manrique, T., Molero, A., Ballesteros, M. A., Morón, I., Gallo, M., \& Fenton, A. A. (2004). Time of day-dependent latent inhibition of conditioned taste aversions in rats. Neurobiology of Learning and Memory, 82, 77-80.

Manzo, L., Donaire, R., Sabariego, M., Papini, M. R., \& Torres, C. (2015). Anti-anxiety self-medication in rats: Oral consumption of chlordiazepoxide and ethanol after reward devaluation. Behavioural Brain Research, 278, 90-97.

McDonald, L. M., Moran, P. M., Vythelingum, G. N., Joseph, M. H., Stephenson, J. D., \& Gray, J. A. (2002). Latent inhibition is attenuated by noise and partially restored by a 5-HT2A receptor antagonist. Behavioural Pharmacology, 13, 663-667.
Melo, L., de Moraes, E., Airoldi, N., \& Sandner, G. (2003). Enhancement of latent inhibition by chronic mild stress in rats submitted to emotional response conditioning. Neural Plasticity, 10, 327-332.

Mitchell, C., \& Flaherty, C. F. (1998). Temporal dynamics of corticosterone elevation in successive negative contrast. Physiology \& Behavior, 64, 287-292.

Molero-Chamizo, A. (2017). Circadian-temporal context and latent inhibition of conditioned taste aversion: Effect of restriction in the intake of the conditioned taste stimulus. Learning \& Behavior, 45, 157163

Mongeau, R., Marcello, S., Sparre, J., Andersen, J., \& Pani, L. (2007). Contrasting effects of diazepam and repeated restraint stress on latent inhibition in mice. Behavioural Brain Research, 183, 147-155.

Mustaca, A. E., \& Papini, M. R. (2005). Consummatory successive negative contrast induces hypoalgesia. International Journal of Comparative Psychology, 18, 333-339.

Ortega, L. A., Glueck, A. C., Daniel, A. M., Prado-Rivera, M. A., White, M. M., \& Papini, M. R. (2014). Memory interfering effects of chlordiazepoxide on consummatory successive negative contrast. Pharmacology, Biochemistry \& Behavior, 116, 96-106.

Ortega, L. A., Solano, J. L., Torres, C., \& Papini, M. R. (2017). Reward loss and addiction: Opportunities for cross-pollination. Pharmacology, Biochemistry, \& Behavior, 154, 39-52.

Overton, D. A. (1974). Experimental methods for the study of state dependent learning. Federation Proceedings, 33, 1800-1813.

Papini, M. R. (2009). Role of opioid receptors in incentive contrast. International Journal of Comparative Psychology, 22, 170 187.

Papini, M. R., \& Dudley, R. T. (1997). Consequences of surprising reward omissions. Review of General Psychology, 1, 175-197.

Papini, M. R., Fuchs, P. N., \& Torres, C. (2015). Behavioral neuroscience of psychological pain. Neuroscience \& Biobehavioral Reviews, 48, 53-69.

Papini, M. R., \& White, N. (1994). Performance during signals for reward omission. Learning \& Motivation, 25, 45-64.

Papini, M. R., Wood, M., Daniel, A. M., \& Norris, J. N. (2006). Reward loss as psychological pain. International Journal of Psychology \& Psychological Therapy, 6, 189-213.

Park, C. R., Zoladz, P. R., Conrad, C. D., Fleshner, M., \& Diamond, D. M. (2008). Acute predator stress impairs the consolidation and retrieval of hippocampus-dependent memory in male and female rats. Learning \& Memory, 15, 271-280.

Pascucci, T., Ventura, R., Latagliata, E. C., Cabib, S., \& PuglisiAllegra, S. (2007). The medial prefrontal cortex determines the accumbens dopamine response to stress through the opposing influences of norepinephrine and dopamine. Cerebral Cortex, 17, 2796-2804

Pearce, J. M., \& Hall, G. (1980). A model for Pavlovian conditioning: Variations in the effectiveness of conditioned but not unconditioned stimuli. Psychological Review, 87, 332-352.

Pecoraro, N., de Jong, H., \& Dallman, M. F. (2009). An unexpected reduction in sucrose concentration activates the HPA axis on successive post shift days without attenuation by discriminative contextual stimuli. Physiology \& Behavior, 96, 651-661.

Pellegrini, S., Wood, M., Daniel, A. M., \& Papini, M. R. (2005). Opioid receptors modulate recovery from consummatory successive negative contrast. Behavioural Brain Research, 164, 239-249.

Rashidy-Pour, A., Sadeghi, H., Taherain, A. A., Vafaei, A. A., \& Fathollahi, Y. (2004). The effects of acute restraint stress and dexamethasone on retrieval of long-term memory in rats: An interaction with opiate system. Behavioural Brain Research, 154, 193-198.

Rau, V., \& Fanselow, M. S. (2009). Exposure to a stressor produces a long lasting enhancement of fear learning in rats. Stress, 12 , $125-133$. 
Shalev, U., Feldon, J., \& Weiner, I. (1998). Latent inhibition is disrupted by acute and repeated administration of corticosterone. International Journal of Neuropsychopharmacology, 1, 103-113.

Smith, S., Fieser, S., Jones, J., \& Schachtman, T. (2008). Effects of swim stress on latent inhibition using a conditioned taste aversion procedure. Physiology \& Behavior, 95, 539-541.

Stout, S., Boughner, R. L., \& Papini, M. R. (2003). Reexamining the frustration effect in rats: Aftereffects of surprising reinforcement and nonreinforcement. Learning \& Motivation, 34, 437-456.
Torres, C., \& Papini, M. R. (2016). Emotional self-medication and addiction. In V. R. Preedy (Ed.), Neuropathology of drug addiction and substance misuse (Vol. 1, pp. 71-81). New York, NY: Elsevier.

Westbrook, R. F., Jones, M. L., Bailey, G. K., \& Harris, J. A. (2000). Contextual control over conditioned responding in a latent inhibition paradigm. Journal of Experimental Psychology: Animal Behavior Processes, 26, 157-173.

Wynne, B., \& Delius, J. D. (1995). Sensitization to apomorphine in pigeons: Unaffected by latent inhibition but still due to classical conditioning. Psychopharmacology, 119, 414 420. 